Received: 15-01-2021

\section{Research Article}

To Cite: Tuluce Demiray S, Bayrakci U, 2021. Soliton Solutions of Generalized Third-Order Nonlinear Schrödinger Equation by Using GKM, Journal of the Institute of Science and Technology, 11(2): 1481-1488.

\title{
Soliton Solutions of Generalized Third-Order Nonlinear Schrödinger Equation by Using GKM Seyma TULUCE DEMIRAY ${ }^{*}$, Ugur BAYRAKCI ${ }^{1}$
}

\begin{abstract}
In this study, we have worked on GKM in order to obtain the soliton solutions of the generalized third-order nonlinear Schrödinger equation. Thus, we have acquired some new soliton solutions of the generalized third-order nonlinear Schrödinger equation which has an important usage area in optical fiber. Also, we have drawn some 2D and 3D surfaces of these obtained results by using Wolfram Mathematica 12. Then, we have shown the validity of the obtained solutions.
\end{abstract}

Keywords: Generalized Kudryashov method, generalized third-order nonlinear Schrödinger equation, soliton solutions

\footnotetext{
${ }^{1}$ Seyma TULUCE DEMIRAY (Orcid ID: 0000-0002-8027-7290), Ugur BAYRAKCI (Orcid ID: 0000-0002-1765-2318), Osmaniye Korkut Ata Üniversitesi, Fen-Edebiyat Fakültesi, Matematik Bölümü, Osmaniye, Türkiye

*Corresponding Author: Seyma TULUCE DEMIRAY, e-mail: seymatuluce@gmail.com
} 


\section{INTRODUCTION}

The nonlinear Schrödinger equations (NLSEs), a category of nonlinear evolution equations (NLEEs), are used in much areas of engineering and applied sciences kind of fluid mechanics, hydrodynamics, applied mathematics, biophysics, optical fibers, mathematical physics, plasma physics, fluid dynamics and so on (Ma, 2019; Liu et al., 2015; Chettouh et al., 2017; Chowdury et al., 2014; Azzouzi et al., 2009; Triki and Taha, 2012; Xu and Zhang, 2007; Triki et al., 2018; Arshad et al., 2017a; Arshad et al., 2017b; Biswas et al., 2017; Seadawy et al., 2018).

Generalized third-order (NLSE), which is a class of the NLSEs, has been the subject of some research recently. Generalized third-order (NLSE) is given as:

$$
i\left(\frac{\partial u}{\partial t}+\frac{\partial^{3} u}{\partial x^{3}}\right)+|u|^{2}\left(\beta_{1} u+i \beta_{2} \frac{\partial u}{\partial x}\right)+i \beta_{3} \frac{\partial\left(|u|^{2}\right)}{\partial x} u=0
$$

Where value of function is a complex and value of coefficients and are real. Generalized thirdorder (NLSE) given by Eq. (1) is an important model which is used to model ultra-short pulses in optical fibers. Solitons and solitary wave solutions of this model have recently been tried to be found by many researchers. Various methods have been studied such as the extended simple equation method and the $\exp (-\phi(\xi))$-expansion method (Lu et al., 2019), the generalized Riccati mapping method (Nasreen et al., 2019), the exp-a function and unified methods (Hosseini et al., 2020), F-expansion method (Seadawy et al., 2020b) and modified extended direct algebraic method (Seadawy et al., 2020a).

Our aim in this article is ascertain the soliton solutions of generalized third-order (NLSE) through GKM (Tuluce Demiray and Bulut, 2015; Pandir et al., 2016; Tuluce Demiray and Bulut, 2016; Tuluce Demiray and Bulut, 2017; Tuluce Demiray and Bulut, 2019). In Section 2, GKM's basic structure is given. In Section 3, some soliton solutions of generalized third-order (NLSE) have been obtained by applying GKM.

\section{MATERIALS AND METHODS}

We take into account a general nonlinear partial differential equation (NLPDE) in the form:

$$
P\left(u, u_{t}, u_{x}, u_{x x}, u_{x t}, \ldots\right)=0 \text {. }
$$

Step1: Firstly, we consider the travelling wave solution as following form;

$$
u(x, y, t)=u(\xi) e^{i P(x, t)}, \xi=k x+w t, P(x, t)=\delta x+\lambda t .
$$

Where $k, w, \delta$ and $\lambda$ arbitrary constants. Equation (2) is turned into ordinary differential equation by Eq. (3):

$$
P\left(u, u^{\prime}, u^{\prime \prime}, u^{\prime \prime \prime}, \ldots\right)=0
$$

Where superscripts denote ordinary derivatives with respect to $\xi$.

Step2: Suppose that we consider the solutions of Eq. (4) as:

$$
u(\xi)=\frac{\sum_{i=0}^{N} a_{i} Z^{i}(\xi)}{\sum_{j=0}^{M} b_{j} Z^{j}(\xi)}=\frac{A[Z(\xi)]}{B[Z(\xi)]}
$$


Where $Z$ is $\frac{1}{1 \pm e^{\xi}}$ 'dir. We should point out that $Z$ is the solution to the following equation.

$$
Z_{\xi}=Z^{2}-Z
$$

Step3: We can ascertain the values of $M$ and $N$ in Eq. (5) through the homogeneous balance principle. Therefore we balance the highest order nonlinear terms in Eq. (4).

Step4: We substitute Eq. (5) into Eq. (4). Thus we obtain a polynomial of $R(Z)$ of $Z$. Then equating the all coefficients of $R(Z)$ to zero, we find an algebraic equation system. By solving this system, we determine $c$ and the variable coefficients of $a_{0}, a_{1}, a_{2}, \ldots, a_{N}, b_{0}, b_{1}, b_{2}, \ldots, b_{M}$. Finally we can obtain the exact solutions of Eq. (4).

\section{Application of GKM to the equation}

To find the traveling wave solutions of Eq. (1) we consider the following transformation:

$$
u(x, y, t)=u(\xi) e^{i P(x, t)}, \xi=k x+w t, P(x, t)=\delta x+\lambda t
$$

Replace Eq. (2) into Eq. (1) and we get the following

$$
3 k^{2} \delta u^{\prime \prime}+\left(\lambda-\delta^{3}\right) u+\left(\delta \beta_{2}+\beta_{1}\right) u^{3}=0 .
$$

And

$$
k^{3} u^{\prime \prime}+\left(w-3 \delta^{2} k\right) u+\frac{k\left(2 \beta_{3}+\beta_{2}\right)}{3} u^{3}=0
$$

By using balance principle in Eq. (9), we obtain

$$
N-M+2=3 N-3 M \Rightarrow N=M+1 .
$$

If we select $M=1$ and $N=2$ we find the following solution

$$
\begin{gathered}
u(\xi)=\frac{a_{0}+a_{1} Z+a_{2} Z^{2}}{b_{0}+b_{1} Z}, \\
u^{\prime}(\xi)=\left(Z^{2}-Z\right)\left[\frac{\left(a_{1}+2 a_{2} Z\right)\left(b_{0}+b_{1} Z\right)-b_{1}\left(a_{0}+a_{1} Z+a_{2} Z^{2}\right)}{\left(b_{0}+b_{1} Z\right)^{2}}\right], \\
u^{\prime \prime}(\xi)=\frac{Z^{2}-Z}{\left(b_{0}+b_{1} Z\right)^{2}}(2 Z-1)\left[\left(a_{1}+2 a_{2} Z\right)\left(b_{0}+b_{1} Z\right)-b_{1}\left(a_{0}+a_{1} Z+a_{2} Z^{2}\right)\right]+\frac{\left(Z^{2}-Z\right)^{2}}{\left(b_{0}+b_{1} Z\right)^{3}} \\
+\left[2 a_{2}\left(b_{0}+b_{1} Z\right)^{2}-2 b_{1}\left(a_{1}+2 a_{2} Z\right)\left(b_{0}+b_{1} Z\right)\right. \\
\left.+2 b_{1}^{2}\left(a_{0}+a_{1} Z+a_{2} Z^{2}\right)\right] .
\end{gathered}
$$

We obtain the soliton solutions of Eq. (1) in the following different cases;

\section{Case1:}

$$
\begin{aligned}
& a_{0}=-\frac{i \sqrt{\frac{3}{2}} k b_{0}}{\sqrt{\beta_{2}+2 \beta_{3}}}, a_{1}=-\frac{a_{2}}{2}+\frac{i \sqrt{6} k b_{0}}{\sqrt{\beta_{2}+2 \beta_{3}}}, b_{1}=-\frac{i a_{2} \sqrt{\beta_{2}+2 \beta_{3}}}{\sqrt{6} k}, \\
& w=\frac{1}{2} k\left(k^{2}+6 \delta^{2}\right) .
\end{aligned}
$$


Substituting the above values in Eq. (11), we acquire the soliton solution of Eq. (1)

$$
u_{1}(x, t)=-\frac{i \sqrt{\frac{3}{2}} k \tanh \left[\frac{1}{2}\left(k x+\frac{1}{2} k t\left(k^{2}+6 \delta^{2}\right)\right)\right]}{\sqrt{\beta_{2}+2 \beta_{3}}} .
$$

$$
u_{2}(x, t)=-\frac{i \sqrt{\frac{3}{2}} k \operatorname{coth}\left[\frac{1}{2}\left(k x+\frac{1}{2} k t\left(k^{2}+6 \delta^{2}\right)\right)\right]}{\sqrt{\beta_{2}+2 \beta_{3}}} .
$$
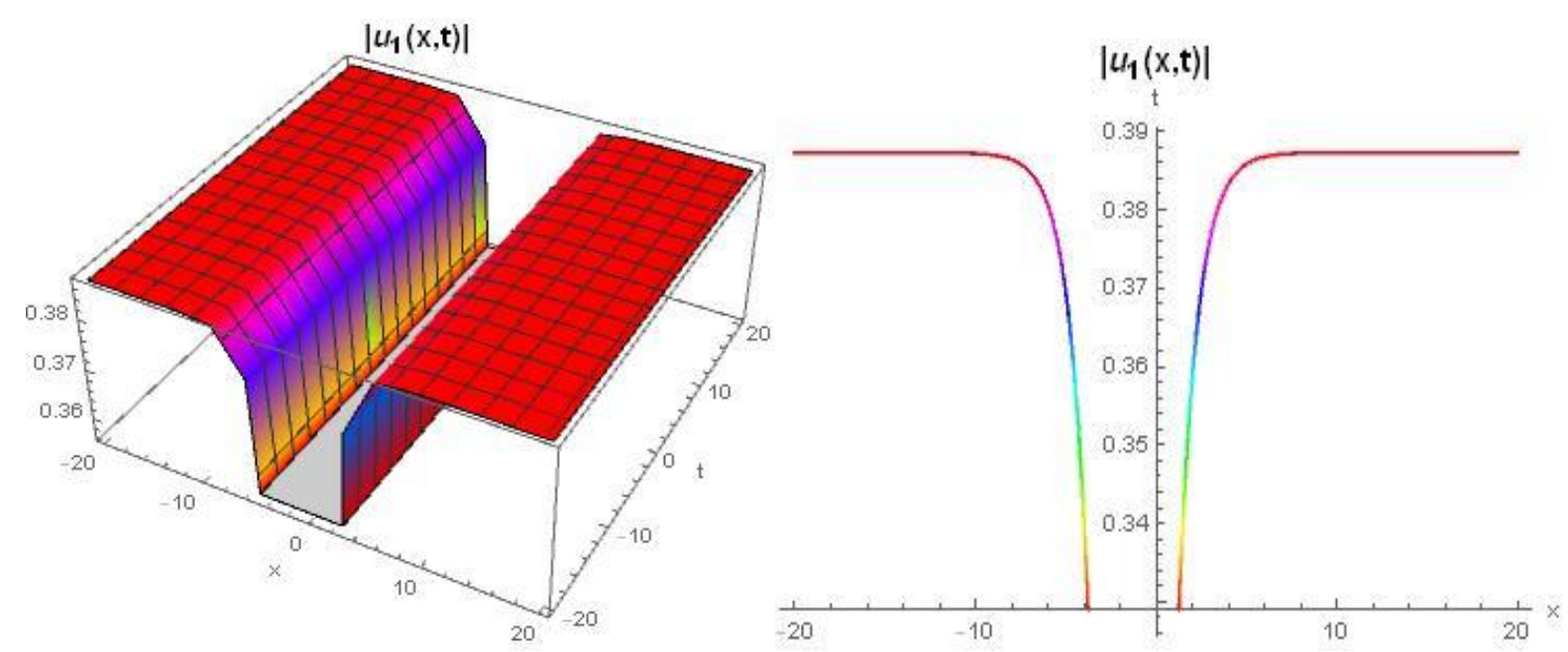

Figure 1: The 3D graph of the solution (15) for $k=1, \delta=2, \beta_{2}=2, \beta_{3}=2,-20<x<20,-20<t<20$ and $2 \mathrm{D}$ graph for this values and $t=0.1$.

\section{Case2:}

$$
\begin{aligned}
& a_{0}=\frac{\sqrt{\frac{3}{2}}\left(-3 k b_{1} \sqrt{-\beta_{2}-2 \beta_{3}}+\sqrt{-k^{2} b_{1}^{2}\left(\beta_{2}+2 \beta_{3}\right)}\right)}{4\left(\beta_{2}+2 \beta_{3}\right)}, a_{1}=-\frac{\sqrt{6} k b_{1}}{\sqrt{-\beta_{2}-2 \beta_{3}}}, a_{2}=\frac{\sqrt{6} k b_{1}}{\sqrt{-\beta_{2}-2 \beta_{3}}}, \\
& b_{0}=-\frac{b_{1}}{2}, w=\frac{1}{4} k\left(5 k^{2}+12 \delta^{2}-\frac{3 k \sqrt{-k^{2} b_{1}^{2}\left(\beta_{2}+2 \beta_{3}\right)}}{b_{1} \sqrt{-\beta_{2}-2 \beta_{3}}}\right) .
\end{aligned}
$$

Substituting the above values in Eq. (11), we acquire the soliton solution of Eq. 


$$
\begin{aligned}
& u_{3}(x, t)=\frac{-\sqrt{3}}{4 \sqrt{2} b_{1}\left(-\beta_{2}-2 \beta_{3}\right)^{3 / 2}}\left(-2 k\left(-1+3 \cosh \left[k x+\frac{1}{4} k t\left(5 k^{2}+12 \delta^{2}-\frac{3 k \sqrt{-k^{2} b_{1}^{2}\left(\beta_{2}+2 \beta_{3}\right)}}{b_{1} \sqrt{\left(-\beta_{2}-2 \beta_{3}\right)}}\right)\right]\right)\right. \\
& \times \operatorname{csch}\left[k x+\frac{1}{4} k t\left(5 k^{2}+12 \delta^{2}-\frac{3 k \sqrt{-k^{2} b_{1}^{2}\left(\beta_{2}+2 \beta_{3}\right)}}{b_{1} \sqrt{\left(-\beta_{2}-2 \beta_{3}\right)}}\right)\right] b_{1}\left(\beta_{2}+2 \beta_{3}\right) \\
& -2 \operatorname{coth}\left[\frac{1}{2}\left(k x+\frac{1}{4} k t\left(5 k^{2}+12 \delta^{2}-\frac{3 k \sqrt{-k^{2} b_{1}^{2}\left(\beta_{2}+2 \beta_{3}\right)}}{b_{1} \sqrt{\left(-\beta_{2}-2 \beta_{3}\right)}}\right)\right)\right] \\
& \left.\quad \times \sqrt{-\beta_{2}-2 \beta_{3}} \sqrt{-k^{2} b_{1}^{2}\left(\beta_{2}+2 \beta_{3}\right)}\right) \cdot \\
& u_{4}(x, t)=\frac{-\sqrt{3}}{4 \sqrt{2} b_{1}\left(-\beta_{2}-2 \beta_{3}\right)^{3 / 2}}\left(-2 k\left(-1+3 \cosh \left[k x+\frac{1}{4} k t\left(5 k^{2}+12 \delta^{2}-\frac{3 k \sqrt{-k^{2} b_{1}^{2}\left(\beta_{2}+2 \beta_{3}\right)}}{b_{1} \sqrt{\left(-\beta_{2}-2 \beta_{3}\right)}}\right)\right]\right)\right. \\
& \quad \times \operatorname{csch}\left[k x+\frac{1}{4} k t\left(5 k^{2}+12 \delta^{2}-\frac{3 k \sqrt{-k^{2} b_{1}^{2}\left(\beta_{2}+2 \beta_{3}\right)}}{b_{1} \sqrt{\left(-\beta_{2}-2 \beta_{3}\right)}}\right)\right] b_{1}\left(\beta_{2}+2 \beta_{3}\right) \\
& \quad \\
& \quad 2 \tanh \left[\frac{1}{2}\left(k x+\frac{1}{4} k t\left(5 k^{2}+12 \delta^{2}-\frac{3 k \sqrt{-k^{2} b_{1}^{2}\left(\beta_{2}+2 \beta_{3}\right)}}{b_{1} \sqrt{\left(-\beta_{2}-2 \beta_{3}\right)}}\right)\right)\right] \\
& \left.\times \sqrt{-\beta_{2}-2 \beta_{3}} \sqrt{-k^{2} b_{1}^{2}\left(\beta_{2}+2 \beta_{3}\right)}\right) .
\end{aligned}
$$
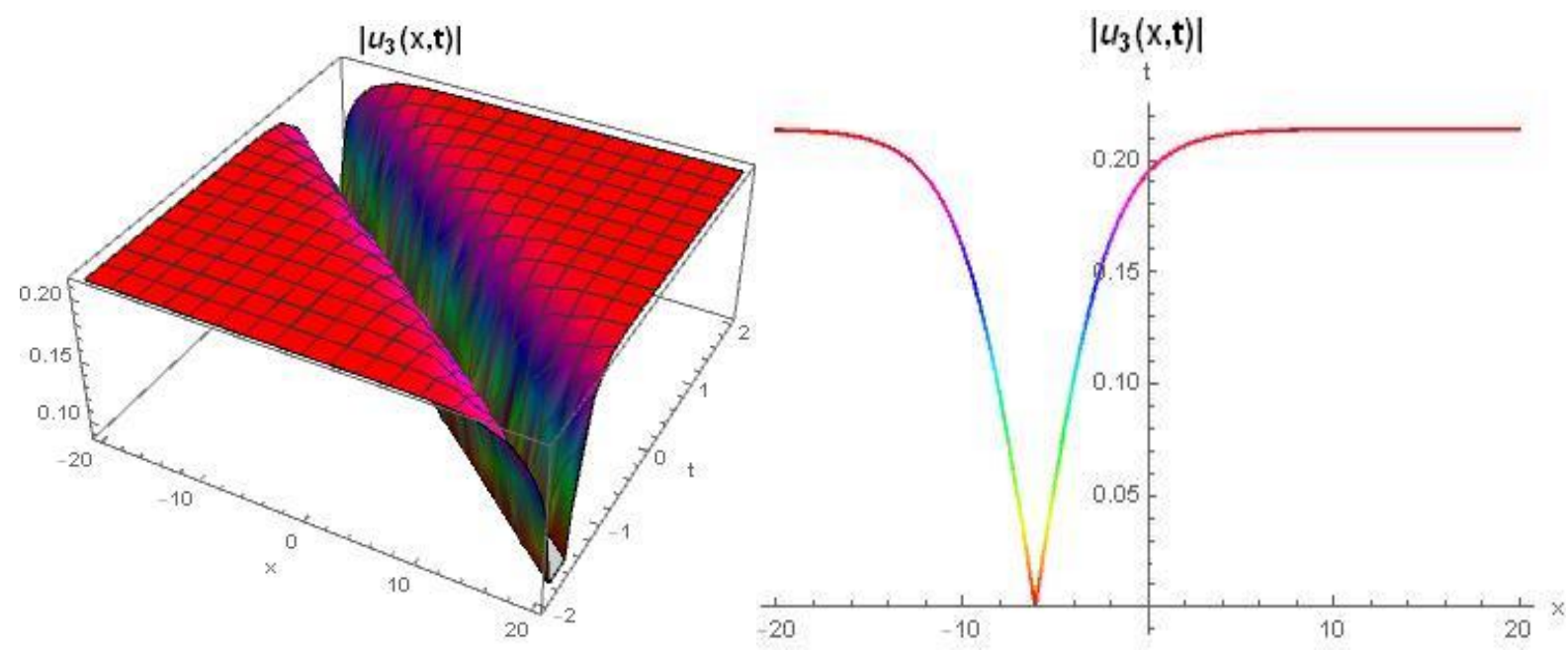

Figure 2: The 3D graph of the solution (18) for $k=0.5, \delta=2, b_{1}=1, \beta_{2}=0.2, \beta_{3}=4,-20<x<20,-2<t<2$ and 2D graph for this values and $t=0.5$.

\section{Case3:}

$$
\begin{aligned}
& a_{0}=-\frac{\sqrt{6} k b_{0}}{\sqrt{-\beta_{2}-2 \beta_{3}}}, a_{1}=\frac{2 \sqrt{6} k b_{0}}{\sqrt{-\beta_{2}-2 \beta_{3}}}, a_{2}=-\frac{2 \sqrt{6} k b_{0}}{\sqrt{-\beta_{2}-2 \beta_{3}}} \\
& b_{1}=-2 b_{0}, w=\left(2 k^{3}+3 k \delta^{2}\right) .
\end{aligned}
$$


Substituting the above values in Eq. (11), we acquire the soliton solution of Eq. (1)

$$
\begin{aligned}
& u_{5}(x, t)=-\frac{\sqrt{6} k \operatorname{coth}\left[k x+t\left(2 k^{3}+3 k \delta^{2}\right)\right]}{\sqrt{-\beta_{2}-2 \beta_{3}}} . \\
& u_{6}(x, t)=-\frac{\sqrt{6} k \tanh \left[k x+t\left(2 k^{3}+3 k \delta^{2}\right)\right]}{\sqrt{-\beta_{2}-2 \beta_{3}}} .
\end{aligned}
$$
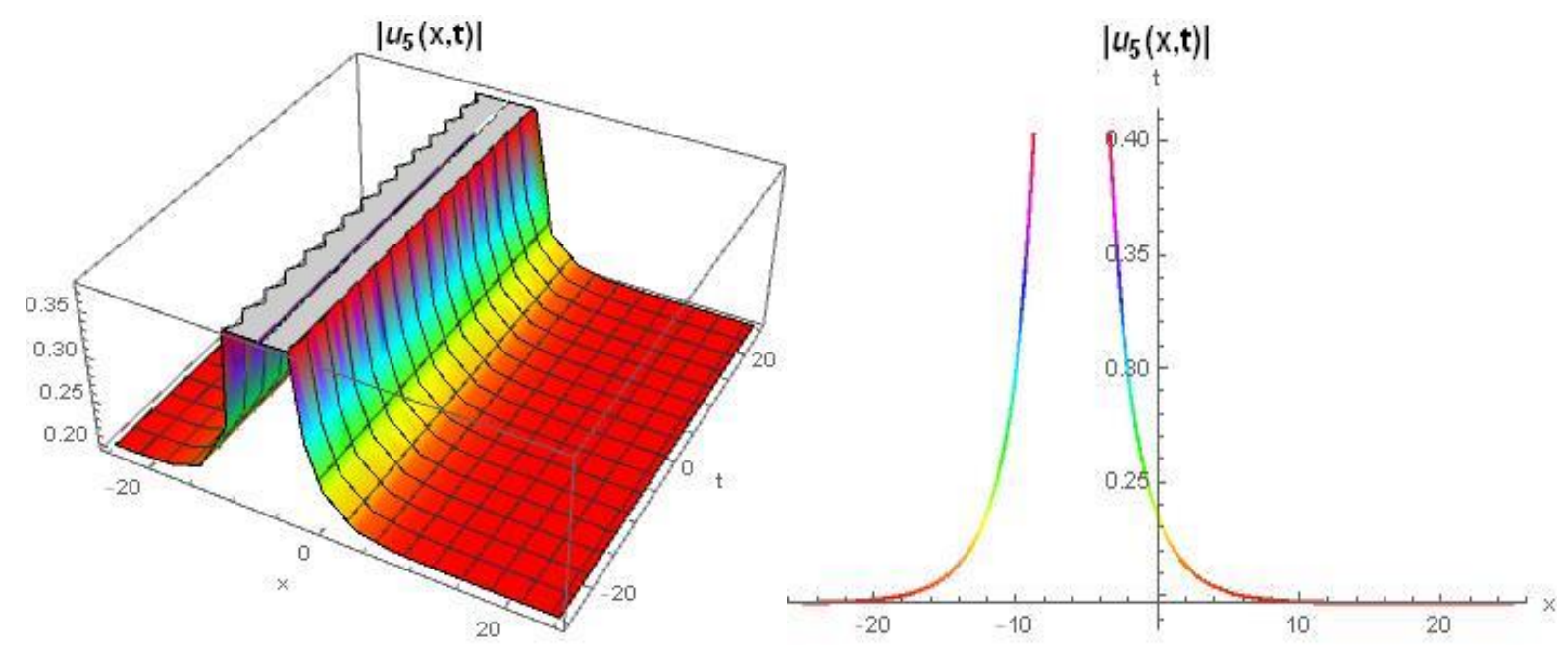

Figure 3: The 3D graph of the solution (21) for $k=0.2, \delta=2, \beta_{2}=0.2, \beta_{3}=3,-25<x<25,-25<t<25$ and $2 \mathrm{D}$ graph for this values and $t=0.5$

\section{RESULTS AND DISCUSSION}

We obtained some soliton solutions of the generalized third-order (NLSE) equation by applying GKM. We proved their accuracy by graphically representing these obtained results by aid of Wolfram Mathematica 12. Several methods were previously applied by some authors to obtain the solutions of the generalized third-order (NLSE) equation. When we check the solutions we found with those of other authors, our (15) and (22) solutions are similar to the (19) and (37) solutions given by Lu et al., the (12) solution given by Nasreen et al. and the (19) solution given by Seadawy et al. In addition to our (16) and (21) solutions are similar to the (17) solution given by Lu et al., the (13) solution given by Nasreen et al. and the (42) solution given by Seadawy et al. According to our research our (18) and (19) solutions are not given before and are new.

\section{CONCLUSION}

In this made study, We obtained the soliton solutions of generalized third-order (NLSE) describing ultra-short pulses in optical fiber. Thus, GKM, which is easier to apply than other methods, is a very effective and reliable method for finding solutions to NLEEs. In addition, the accuracy of the obtained solutions has been shown with graphical representations.

\section{Conflict of Interest}

The article authors declare that there is no conflict of interest between them.

\section{Author's Contiıbutions}

The authors declare that they have contributed equally to the article. 


\section{REFERENCES}

Arshad M, Seadawy AR, Lu D, 2017a. Bright-dark solitary wave solutions of generalized higher-order nonlinear Schrödinger equation and its application in optics. Journal of Electromagnetic Waves and Applications, 31(16): 1711-1721.

Arshad M, Seadawy AR, Lu D, 2017b. Exact bright-dark solitary wave solutions of the higher-order cubicquintic nonlinear Schrödinger equation and its stability. Optik-International Journal for Light and Electron Optics, 138: 40-49.

Azzouzi F, Triki H, Mezghiche K, El Akrmi A, 2009. Solitary wave solutions for high dispersive cubicquintic nonlinear Schrödinger equation. Chaos Solitons and Fractals, 39(3): 1304-1307.

Biswas A, Triki H, Zhou Q, Moshokoa SP, Ullah MZ, Belic M, 2017. Cubic-quartic optical solitons in Kerr and power law media. Optik-International Journal for Light and Electron Optics, 144: 357-362.

Chettouh S, Triki H, El-Akrmi Zhou AQ, Moshokoa SP, Ullah MZ, Biswas A, Belic M, 2017. Dipole solitons in an extended nonlinear Schrödinger's equation with higher-order even and odd terms. OptikInternational Journal for Light and Electron Optics, 145: 644-649.

Chowdury A, Kedziora DJ, Ankiewicz A, Akhmediev N, 2014. Soliton solutions of an integrable nonlinear Schrodinger equation with quintic terms. Physical Review, 90(032922): 1-9.

Hosseini K, Osman MS, Mirzazadeh M, Rabiei F, 2020. Investigation of different wave structures to the generalized third-order nonlinear Scrödinger equation. Optik-International Journal for Light and Electron Optics, 206(164259): 1-7.

Liu W, Qiu DQ, He JS, 2015. Localized properties of rogue wave for a higher-order nonlinear Schrödinger equation. Communications in Theoretical Physics, 63(5): 525-534.

Lu D, Seadawy AR, Wang J, Arshad M, Farooq U, 2019. Soliton solutions of the generalised third-order nonlinear Schrödinger equation by two mathematical methods and their stability. Pramana Journal of Physics, 93(44): 1-9.

Ma YL, 2019. Interaction and energy transition between the breather and rogue wave for a generalized nonlinear Schrödinger system with two higher-order dispersion operators in optical fibers. Nonlinear Dynamics, 97(1): 95-105.

Nasreen N, Seadawy AR, Lu D, Albarakati WA, 2019. Dispersive solitary wave and soliton solutions of the gernalized third order nonlinear Schrödinger dynamical equation by modified analytical method. Results in Physics, 15(102641): 1-7.

Pandir Y, Tuluce Demiray S, Bulut H, 2016. A new approach for some NLDEs with variable coefficients. Optik-International Journal for Light and Electron Optics, 127(23): 11183-11190.

Seadawy AR, Kumar D, Chakrabarty AK, 2018. Dispersive optical soliton solutions for the hyperbolic and cubic-quintic nonlinear Schrödinger equations via the extended sinh-Gordon equation expansion method. European Physical Journal Plus, 133(182): 1-11.

Seadawy AR, Arshad M, Lu D, 2020a. The weakly nonlinear wave propagation of the generalized thirdorder nonlinear Schrödinger equation and its applications. Waves in Random and Complex Media, 113.

Seadawy AR, Nasreen N, Lu D, 2020b. Complex model ultra-short pulses in optical fibers via generalized third-order nonlinear Schrödinger dynamical equation. International Journal of Modern Physics B, 34(17): 1-9.

Triki H, Taha TR, 2012. Solitary wave solutions for a higher order nonlinear Schrödinger equation. Mathematics and Computers in Simulation, 82(7): 1333-1340.

Triki H, Choudhuri A, Porsezian K, Dinda PT, 2018. Dark solitons in an extended nonlinear Schrödinger equation with higher-order odd and even terms. Optik-International Journal for Light and Electron Optics, 164: 661-670.

Tuluce Demiray S, Bulut H, 2015. New exact solutions of the New Hamiltonian Amplitude-Equation and Fokas Lenells equation. Entropy, 17(9): 6025-6043. 
Tuluce Demiray S, Bulut H, 2016. Generalized Kudryashov method for nonlinear fractional double sinhpoisson equation. Journal of Nonlinear Science and Applications, 9(3): 1349-1355.

Tuluce Demiray S, Bulut H, 2017. New soliton solutions of Davey-Stewartson equation with power-law nonlinearity. Optical and Quantum Electronics, 49(117): 1-8.

Tuluce Demiray S, Bulut H, 2019. Soliton solutions of some non-linear evolution problems by GKM. Neural Computing and Applications, 31(1): 287-294.

Xu LP, Zhang JL, 2007. Exact solutions to two higher order nonlinear Schrodinger equations. Chaos Solitons and Fractals, 31(4): 937-942. 